\title{
FERTILITY STUDIES IN THE FIELD BEAN (VICIA FABA L.)
}

\author{
II. INBREEDING \\ D. G. ROWLANDS \\ Welsh Plant Breeding Station, Aberystwyth *
}

\section{INTRODUCTION}

Received 3.vi.6I

Previous work has indicated that Vicia faba is a naturally crosspollinating species but under field conditions insect pollination appears to be inadequate and much of the seed that is set (up to 70 per cent. of the total) results from self-pollination (Rowlands, 1958, 1960). Moreover, the average proportion of flowers that set in the field is 20-25 per cent., which suggests that even self-pollination is not very successful, in fact data from controlled self-pollination indicate considerable self-sterility.

A hypothesis was put forward in the first paper to explain this self-sterility in terms of homozygosity of deleterious recessive genes which can accumulate in any outbreeding system.

Breakdown of the original outbreeding system resulting from migration of the species from its centre of origin and cultivation practices, coupled with unsatisfactory insect pollination, could be followed by zygotic sterility caused by deleterious recessive genes. The present system is, therefore, one of balance where the pressure to inbreed is counteracted by the requirement for "tripping" of the flowers and zygotic infertility.

From the point of view of yield improvement it would appear that reliance on insect pollination for seed production introduces too much uncertainty, but in common with other cross-fertilising plants, inbreeding in beans results in loss of vigour. Nevertheless for ease of handling, a self-tripping, self-fertile, line would offer great advantages. Inbreeding in field beans is therefore here discussed in relation to the use of pure lines in improvement work.

\section{PRELIMINARY STUDIES}

The use of a white-flowered line (Ch I 7o) of field beans enabled a comparison of hybrids and inbreds to be made. Alternate rows of the white-flowered (recessive) and coloured-flowered (dominant) lines were grown and the progeny from the white-flowered line separated into outcrossed or inbred types in the subsequent year. (The whiteflowered line originated from a single plant and was already inbred.) The distribution of flower number and pod number per plant in the two groups of material under field conditions is shown in fig. I.

* Now at Unilever Research Laboratory, Colworth House, Bedford. 
In the case of both flowers and pods, a wide variation characterises the outcrosses while this variation is much reduced in the inbreds. Moreover, mean flower and pod number is lower in the inbred than in

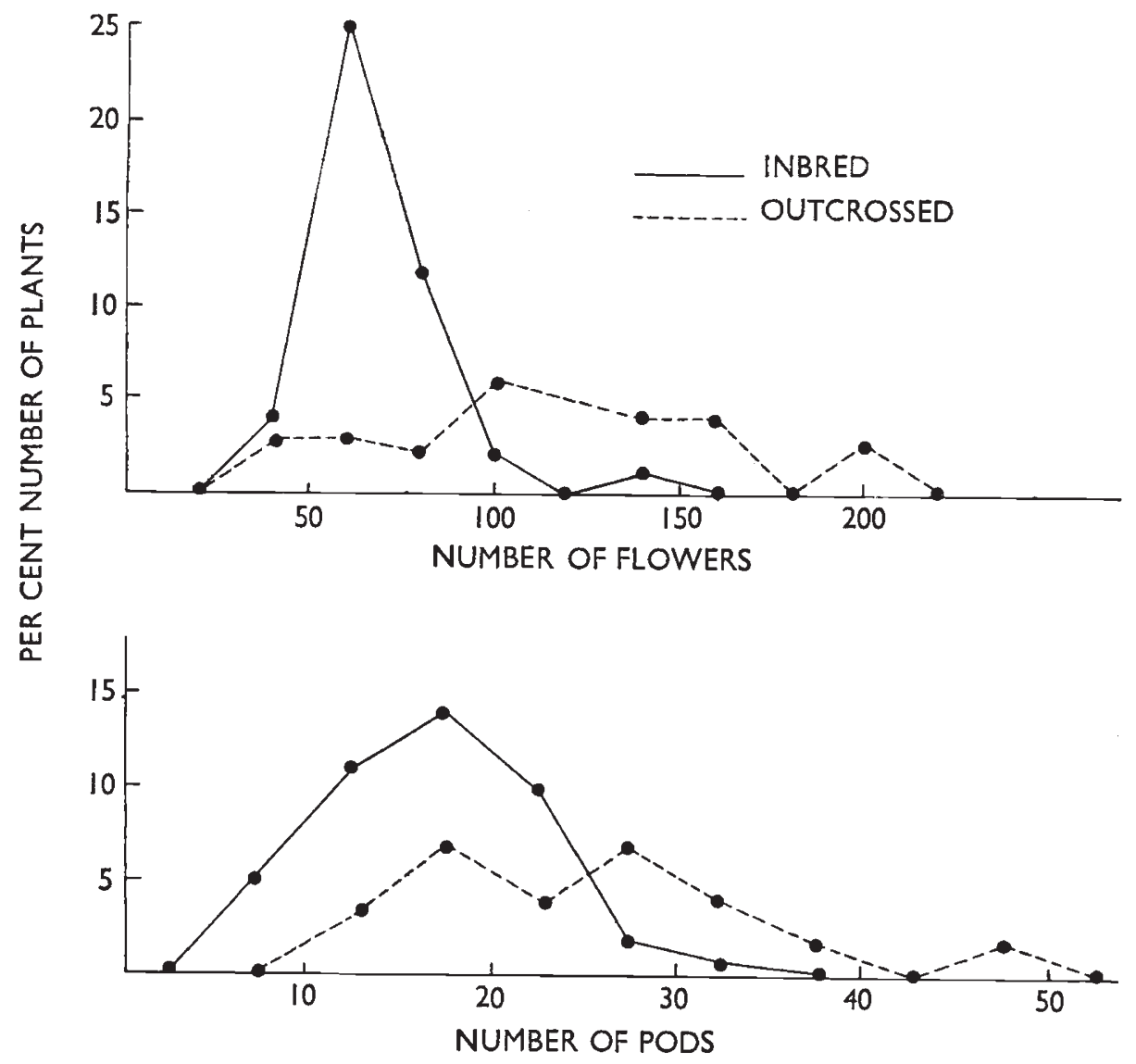

FIG. I.-Frequency distribution of flowers and pods per plant in inbred and outcrossed bean plants.

the crossbred material. Some of the inbred material did, however, produce more than 20 pods per plant and this suggested further investigation into the behaviour of pod setting during inbreeding.

\section{GENERAL INBREEDING STUDIES IN THE GLASSHOUSE}

Material was selected for study from six field populations namely:

Ch 170 the white-flowered already inbred line

Ch 4 a commercial stock of tick beans

B 26 a selection from $\mathrm{Ch} 4$

Ch $103 / 2$ a selection from a commercial line (ex Dorset)

Ch $35 / 2$ a selection from a commercial line (ex Devon)

Cp 145 a high-yielding flower colour selection. 
Two samples of five plants were taken from each of the populations, one comprising five very good podding plants, and the other, five plants taken at random. In the case of Ch I 70 five additional good plants were taken (A selections) and from the $\mathrm{Ch} 4$ population five of the worst podding plants that could be found. The pod numbers of each of these parent plants are shown in table $\mathrm{I}$.

TABLE I

Pod numbers of parent plants for inbreeding experiment (open-pollination, 1955)

\begin{tabular}{|c|c|c|c|c|c|c|c|c|c|c|}
\hline & \multirow{2}{*}{ Population } & & & & \multicolumn{5}{|c|}{ Plant reference no. } & \multirow{2}{*}{ Mean } \\
\hline & & & & & I & 2 & 3 & 4 & 5 & \\
\hline $\mathrm{Ch}$ I 70 & $\begin{array}{l}\text { Selected } \\
\text { A Selection } \\
\text { Random * }\end{array}$ & . & . & & $\begin{array}{l}78 \\
55 \\
\cdots\end{array}$ & $\begin{array}{l}70 \\
50 \\
\cdots\end{array}$ & $\begin{array}{l}79 \\
48 \\
\cdots\end{array}$ & $\begin{array}{l}64 \\
5^{1} \\
\cdots\end{array}$ & $\begin{array}{r}\text { I I } 7 \\
6 \text { I } \\
\ldots\end{array}$ & $\begin{array}{l}8 \mathrm{I} \cdot 6 \\
53 \cdot 0 \\
\ldots\end{array}$ \\
\hline $\mathrm{Ch}_{4}$ & $\begin{array}{l}\text { Selected } \\
\text { Random } \\
\text { Worst }\end{array}$ & . & . & . & $\begin{array}{l}40 \\
3 \text { I } \\
\text { I I }\end{array}$ & $\begin{array}{l}39 \\
24 \\
\text { I } 3\end{array}$ & $\begin{array}{l}35 \\
24 \\
\text { I I }\end{array}$ & $\begin{array}{l}35 \\
\text { I } 5 \\
\text { I I }\end{array}$ & $\begin{array}{l}32 \\
21 \\
14\end{array}$ & $\begin{array}{l}36 \cdot 2 \\
23 \cdot 0 \\
12 \cdot 0\end{array}$ \\
\hline B 26 & $\begin{array}{l}\text { Selected } \\
\text { Random }\end{array}$ & . & . & - & $\begin{array}{l}29 \\
13\end{array}$ & $\begin{array}{l}44 \\
24\end{array}$ & $\begin{array}{l}36 \\
12\end{array}$ & $\begin{array}{r}33 \\
9\end{array}$ & $\begin{array}{l}70 \\
30\end{array}$ & $\begin{array}{l}42 \cdot 4 \\
17 \cdot 6\end{array}$ \\
\hline $\mathrm{Ch}{ }_{10} / 2$ & $\begin{array}{l}\text { Selected } \\
\text { Random }\end{array}$ & . & . & . & $\begin{array}{l}37 \\
33\end{array}$ & $\begin{array}{r}29 \\
9\end{array}$ & $\begin{array}{l}43 \\
14\end{array}$ & $\begin{array}{l}5^{6} \\
23\end{array}$ & $\begin{array}{r}40 \\
8\end{array}$ & $\begin{array}{l}41 \cdot 0 \\
17 \cdot 4\end{array}$ \\
\hline Ch $35 / 2$ & $\begin{array}{l}\text { Selected } \\
\text { Random }\end{array}$ & . & . & . & $\begin{array}{l}27 \\
26\end{array}$ & $\begin{array}{l}31 \\
23\end{array}$ & $\begin{array}{l}38 \\
10\end{array}$ & $\begin{array}{l}28 \\
15\end{array}$ & $\begin{array}{l}43 \\
\text { I I }\end{array}$ & $\begin{array}{l}33 \cdot 4 \\
17 \cdot 0\end{array}$ \\
\hline Cp I 45 & $\begin{array}{l}\text { Selected } \\
\text { Random* }\end{array}$ & • & . & . & $\begin{array}{l}29 \\
\cdots\end{array}$ & $\begin{array}{l}42 \\
\cdots\end{array}$ & $\begin{array}{l}28 \\
\ldots\end{array}$ & $\begin{array}{l}45 \\
\cdots\end{array}$ & $\begin{array}{l}42 \\
\cdots\end{array}$ & $\begin{array}{c}37 \cdot 2 \\
\ldots\end{array}$ \\
\hline
\end{tabular}

* Pod numbers were not recorded for random plants of $\mathrm{Ch}$ I 70 and $\mathrm{Cp}$ I 45.

Five progeny plants from each original parent were grown in an insect-proof glasshouse during $195^{6}$ and pod and seed number per plant recorded on each individual. As many flowers as possible were artificially manipulated to simulate the effect of tripping, so that the data obtained reflected the capacity of each plant to set selfed seed.

The most significant point in table 2 is the low pod-setting on selfing as compared with the performance of the parental lines under open-pollination. This progeny performance on selfing is not correlated with the parental pod set, and their self-fertilities are all of the same order. In general, the selected material would appear to possess lower self-fertilities than the random material. This is to be expected if the selected parents are highly podding by virtue of heterozygosity for self-sterility genes, and this superiority would not be apparent in their progeny. Despite the rather low mean pod number per plant, most of the lines contained occasional plants producing I 5-20 pods, which may be regarded as an adequate set in the field. Seed production 
per plant, and also the mean number of seeds per pod, are reduced, selected plants again suffering more than random plants.

TABLE 2

Mean pod and seed setting of parental lines and $S_{0}$ segregates from each ( 1956$)$

\begin{tabular}{|c|c|c|c|c|c|c|c|}
\hline & & \multirow{2}{*}{$\begin{array}{l}\text { Parental pod } \\
\text { mean (open- } \\
\text { pollination) }\end{array}$} & \multicolumn{3}{|c|}{$\begin{array}{l}\text { Pods per plant on selfing } \\
\mathrm{S}_{0} \text { segregates }\end{array}$} & \multicolumn{2}{|c|}{ Seeds per } \\
\hline & & & Mean & Range & $\begin{array}{l}\text { Per cent. of } \\
\text { parent }\end{array}$ & Plant & Pod \\
\hline $\mathrm{Ch}_{1} 7^{\circ}$ & $\begin{array}{l}\text { Selected } \\
\text { A Selection } \\
\text { Random . }\end{array}$ & $\begin{array}{c}8 I \cdot 6 \\
53 \cdot 0 \\
\ldots\end{array}$ & $\begin{array}{l}7 \cdot 2 \\
9 \cdot 7 \\
5 \cdot 6\end{array}$ & $\begin{array}{l}0-20 \\
4-22 \\
0-28\end{array}$ & $\begin{array}{c}8 \cdot 8 \\
I 8 \cdot 3 \\
\ldots\end{array}$ & $\begin{array}{c}14 \cdot 2 \\
\ldots \\
12 \cdot 3\end{array}$ & $\begin{array}{l}I \cdot 9 \\
\ldots \\
2 \cdot 2\end{array}$ \\
\hline Ch $103 / 2$ & $\begin{array}{l}\text { Selected . } \\
\text { Random . }\end{array}$ & $\begin{array}{l}4 I \cdot 0 \\
\text { I } 7 \cdot 4\end{array}$ & $\begin{array}{r}9 \cdot 8 \\
\text { I0.2 }\end{array}$ & $\begin{array}{l}0-29 \\
0-26\end{array}$ & $\begin{array}{l}23 \cdot 9 \\
58 \cdot 6\end{array}$ & $\begin{array}{l}20 \cdot 4 \\
21 \cdot 6\end{array}$ & $\begin{array}{l}R \cdot I \\
2 \cdot I\end{array}$ \\
\hline Ch $35 / 2$ & $\begin{array}{l}\text { Selected } \\
\text { Random }\end{array}$ & $\begin{array}{l}33 \cdot 4 \\
\text { I } 7 \cdot 0\end{array}$ & $\begin{aligned} 7 \cdot 0 \\
10 \cdot 0\end{aligned}$ & $\begin{array}{l}2-28 \\
4-23\end{array}$ & $\begin{array}{l}21 \cdot 0 \\
58 \cdot 8\end{array}$ & $\begin{array}{l}14 \cdot 7 \\
20 \cdot 6\end{array}$ & $\begin{array}{l}2 \cdot I \\
2 \cdot I\end{array}$ \\
\hline B 26 & $\begin{array}{l}\text { Selected } \\
\text { Random }\end{array}$ & $\begin{array}{l}42 \cdot 4 \\
17 \cdot 6\end{array}$ & $\begin{array}{l}6 \cdot 7 \\
7 \cdot 7\end{array}$ & $\begin{array}{l}0=13 \\
I=I 5\end{array}$ & $\begin{array}{l}\text { I } 5 \cdot 8 \\
43 \cdot 8\end{array}$ & $\begin{array}{l}\text { I } 1 \cdot 8 \\
\text { I } 4 \cdot 9\end{array}$ & $\begin{array}{l}I \cdot 8 \\
I \cdot 9\end{array}$ \\
\hline $\mathrm{Ch}_{4}$ & $\begin{array}{l}\text { Selected } \\
\text { Random } \\
\text { Worst }\end{array}$ & $\begin{array}{l}36 \cdot 2 \\
23 \cdot 0 \\
12 \cdot 0\end{array}$ & $\begin{array}{l}7 \cdot 8 \\
8 \cdot 1 \\
6 \cdot 2\end{array}$ & $\begin{array}{l}I-I 9 \\
O-20 \\
I-I 2\end{array}$ & $\begin{array}{l}2 I \cdot 5 \\
35 \cdot 2 \\
5 I \cdot 7\end{array}$ & $\begin{array}{l}I 2 \cdot I \\
I 5 \cdot I \\
14 \cdot 3\end{array}$ & $\begin{array}{l}\text { I } \cdot 6 \\
I \cdot 9 \\
2 \cdot 3\end{array}$ \\
\hline Cp I 45 & $\begin{array}{l}\text { Selected } \\
\text { Random }\end{array}$ & $\begin{array}{c}37^{\cdot 2} \\
\cdots\end{array}$ & $\begin{array}{l}6 \cdot 0 \\
6 \cdot 6\end{array}$ & $\begin{array}{ll}0 \\
0-10 \\
I-2 I\end{array}$ & $\begin{array}{c}\text { I } 6 \cdot I \\
\cdots\end{array}$ & $\begin{array}{l}\text { I } 5 \cdot 0 \\
\text { I } 5 \cdot 3\end{array}$ & $\begin{array}{l}2 \cdot 5 \\
2 \cdot 3\end{array}$ \\
\hline Mean & $\cdot \quad \cdot$ & $34 \cdot 3$ & $7 \cdot 8$ & $\cdots$ & $3^{I \cdot I}$ & $15 \cdot 6$ & $2 \cdot I$ \\
\hline
\end{tabular}

From this population, those plants bearing 20 or more pods or more seeds per plant were selected. Fifty seeds of each of the eight highest lines were sown, together with five seeds of each of seventeen fairly good lines. In addition, five seeds of each of the 22 progenies of $\mathrm{Ch} 4$ were sown. These plants were allowed to set seed without the aid of " tripping" and an estimate of spontaneous self-fertility was obtained. These results are presented in table 3 .

Pod number per plant is lower than the corresponding progeny mean in 1956 , and very much lower than in the selected parental $S_{0}$ plants. Seed number per plant is reduced and so is the percentage of flowers setting pods, and the mean seed number per roo flowers. Some of the progenies contain a high proportion of sterile plants; Ch 103/2 progenies in general were worse than the others, and Ch $103 /$ ${ }_{2} R_{3} / 2$ were worst of all, with over $5^{\circ}$ per cent. of the plants sterile. Least affected by inbreeding is Ch I $70 \mathrm{R}_{5 / 2}$ with fewest sterile plants and highest pod and seed sets. The family $\mathrm{Ch}_{\mathrm{I}} \mathrm{7O}$ is based on a single white-flowered plant and is already somewhat inbred. Ch I $70 \mathrm{R}_{5 / 2}$, Ch 35/2 R 3/1 and Ch 103/2 S 2/4 are perhaps the most profitable progenies to follow up in attempting to select for self-fertility. 
TABLE 3

Mean self-fertility of $S_{1}$ progenies from highly self-fertile $S_{0}$ plants (1957)

\begin{tabular}{|c|c|c|c|c|c|c|c|c|c|c|}
\hline \multirow{2}{*}{ Progeny } & & \multirow{2}{*}{$\begin{array}{l}\text { No. of } \\
\text { plants } \\
\text { grown }\end{array}$} & \multirow{2}{*}{$\begin{array}{l}\text { No. of } \\
\text { pods } \\
\text { on } S_{0} \\
\text { plants }\end{array}$} & \multirow{2}{*}{$\begin{array}{c}\text { Per } \\
\text { cent. } \\
\text { sterile } \\
\text { plants }\end{array}$} & \multicolumn{3}{|c|}{$\begin{array}{c}\text { Mean no. per } \\
\text { plant of }\end{array}$} & \multirow{2}{*}{$\begin{array}{c}\text { Mean } \\
\text { no. of } \\
\text { seeds } \\
\text { per } \\
\text { pod }\end{array}$} & \multirow{2}{*}{$\begin{array}{l}\text { Per } \\
\text { cent. } \\
\text { flowers } \\
\text { forming } \\
\text { pods }\end{array}$} & \multirow{2}{*}{$\begin{array}{l}\text { Seeds } \\
\text { per } \\
\text { Ioo } \\
\text { flowers }\end{array}$} \\
\hline & & & & & Flowers & Pods & Seeds & & & \\
\hline $\operatorname{Ch}_{170} \mathrm{R}_{5 / 2}$ & - & 32 & 28 & 3 & 125 & $5 \cdot 7$ & $9 \cdot 6$ & $I \cdot 7$ & $4 \cdot 6$ & $7 \cdot 7$ \\
\hline Ch $35 / 2 R_{3 / 1}$ & - & 44 & 23 & 5 & I I 9 & $4 \cdot 8$ & $7 \cdot 5$ & $I \cdot 6$ & $4^{\circ} 0$ & $6 \cdot 3$ \\
\hline Ch $35 / 2 R_{4 / I}$ & - & 43 & 22 & 14 & I 27 & $4 \cdot 3$ & $6 \cdot 0$ & $I \cdot 4$ & $3 \cdot 4$ & $4 \cdot 7$ \\
\hline Ch $35 / 2 \mathrm{~S}_{3} / 2$ & . & 49 & 28 & 16 & I 25 & $3 \cdot 9$ & $5 \cdot I$ & $I \cdot 3$ & $3 \cdot 1$ & $4 \cdot 2$ \\
\hline $\mathrm{Ch}_{103 / 2 \mathrm{R}_{\mathrm{I}} / \mathrm{I}}$ & . & 37 & $2 I$ & 27 & 140 & $2 \cdot 4$ & $2 \cdot 4$ & $I \cdot 0$ & $I \cdot 6$ & $5 \cdot 6$ \\
\hline $\mathrm{Ch}$ I $03 / 2 R_{4} / \mathrm{I}$ & . & $4^{I}$ & 26 & 32 & I 33 & $2 \cdot I$ & $2 \cdot 5$ & $I \cdot 2$ & $I \cdot 6$ & I $\cdot 9$ \\
\hline Ch I $03 / 2 R_{3} / 2$ & . & $3^{8}$ & 24 & $5^{8}$ & 98 & $3 \cdot 9$ & $I \cdot 0$ & $0 \cdot 3$ & $4 \cdot 0$ & $I \cdot O$ \\
\hline $\mathrm{Ch}_{1} \mathrm{O}_{3} / 2 \mathrm{~S}_{2} / 4$ & . & 42 & 29 & 17 & IO9 & $5 \cdot 4$ & $9 \cdot I$ & $1 \cdot 7$ & $5 \cdot 0$ & $8 \cdot 4$ \\
\hline Mean & - & $\cdots$ & $25^{\cdot I}$ & $2 I \cdot 5$ & 122 & $4 \cdot I$ & $5 \cdot 4$ & $I \cdot 3$ & $3 \cdot 4$ & $4 \cdot 4$ \\
\hline
\end{tabular}

TABLE 4

Pod and seed setting in $S_{1}$ progeny plants and $S_{2}$ families of Ch $170 R_{5 / 2}\left(195^{8}\right)$

\begin{tabular}{|c|c|c|c|c|c|c|}
\hline \multirow{3}{*}{ Progeny no. } & \multicolumn{3}{|c|}{$S_{1}$ progeny plants } & \multicolumn{3}{|c|}{$\mathrm{S}_{2}$ family means } \\
\hline & \multicolumn{3}{|c|}{ No. of } & \multicolumn{3}{|c|}{ No. of } \\
\hline & Pods & Seeds & $\begin{array}{l}\text { Seeds per } \\
\text { pod }\end{array}$ & Pods & Seeds & $\begin{array}{l}\text { Seeds per } \\
\text { pod }\end{array}$ \\
\hline I & 3 & 4 & $\mathrm{I} \cdot 3$ & I 2.5 & $21 \cdot 5$ & $1 \cdot 7$ \\
\hline 2 & 18 & 35 & I 9 & $2 \cdot 4$ & $4^{\cdot 2}$ & I. 8 \\
\hline 3 & $I$ & 2 & $2 \cdot 0$ & $2 \cdot 0$ & 3.5 & I 8 \\
\hline 4 & 6 & 9 & $1 \cdot 5$ & $8 \cdot 0$ & $170^{\circ}$ & $2 \cdot I$ \\
\hline 5 & 2 & 2 & I'o & 4.5 & $8 \cdot 0$ & $\mathrm{I} .8$ \\
\hline 6 & 6 & I I & I 8 & 50 & $7 \cdot 8$ & I. 6 \\
\hline 7 & 2 & 4 & $2 \cdot 0$ & $6 \cdot 0$ & $11 \cdot 7$ & $2 \cdot 0$ \\
\hline 8 & I 4 & 17 & $1 \cdot 2$ & $5 \cdot 0$ & $8 \cdot 0$ & $1 \cdot 6$ \\
\hline 9 & I0 & 17 & 1.7 & $9 \cdot 3$ & $19 \cdot 7$ & $2 \cdot I$ \\
\hline I I & 1 & $I$ & I 0 & $2 \cdot 7$ & $4 \cdot 3$ & I. 6 \\
\hline 13 & 9 & 18 & $2 \cdot 0$ & $9 \cdot 5$ & $16 \cdot 0$ & $1 \cdot 7$ \\
\hline 14 & 5 & 5 & I $\cdot 0$ & 8.0 & $12 \cdot 7$ & I. 6 \\
\hline 16 & 6 & 8 & $1 \cdot 3$ & 0.5 & 0.5 & I'O \\
\hline 17 & 4 & 8 & $2 \cdot 0$ & $2 \cdot 3$ & $4^{\circ} 0$ & $1 \cdot 7$ \\
\hline 18 & 7 & I 5 & $2 \cdot I$ & $3 \cdot 3$ & $6 \cdot 0$ & 1.8 \\
\hline 20 & 4 & 7 & I 8 & 6.5 & I $6 \cdot 0$ & $2 \cdot 5$ \\
\hline 21 & $\begin{array}{l}16 \\
16\end{array}$ & 27 & $1 \cdot 7$ & $10 \%$ & $17^{\circ} 0$ & 1.7 \\
\hline 22 & 9 & 15 & 1.7 & $3 \cdot 3$ & $6 \cdot 8$ & $2 \cdot I$ \\
\hline 23 & 5 & II & $2 \cdot 2$ & 30 & $6 \cdot 3$ & $2 \cdot I$ \\
\hline 24 & 3 & 8 & $2 \cdot 7$ & $17 \cdot 0$ & $34^{\circ}$ & $2 \cdot 0$ \\
\hline 25 & 7 & 10 & I. 4 & 15.0 & $32 \cdot 0$ & $2 \cdot I$ \\
\hline 26 & 3 & $\mathrm{I}$ & 0.3 & $9^{\circ} 0$ & $10 \cdot 0$ & $I \cdot 1$ \\
\hline 27 & 3 & 3 & I.O & $2 \cdot 0$ & $3 \cdot 5$ & 1.8 \\
\hline 28 & 4 & I0 & $2 \cdot 5$ & 5.4 & 10.6 & $2 \cdot 0$ \\
\hline 29 & I & 2 & $2 \cdot 0$ & $18 \cdot 0$ & $3^{8 \cdot 0}$ & $2 \cdot 1$ \\
\hline 30 & 5 & 6 & $1 \cdot 2$ & $11 \cdot 5$ & I 8.0 & $1 \cdot 6$ \\
\hline $3 \mathrm{I}$ & II & 19 & $1 \cdot 7$ & $7 \cdot 3$ & II 0 & $1 \cdot 5$ \\
\hline $3^{2}$ & 2 & 5 & $2 \cdot 5$ & $6 \cdot 3$ & I $4 \cdot 3$ & $2 \cdot 3$ \\
\hline Mean . & 5.9 & $10 \cdot 0$ & $I \cdot 7$ & $5 \cdot 9$ & II $\cdot O$ & $1 \cdot 8$ \\
\hline
\end{tabular}


In contrast to the above material the less highly fertile $S_{0}$ progenies were only scored for pod and seed number but the data were comparable with those obtained from the more highly fertile $\mathrm{S}_{0}$ plants.

Progenies from $\mathrm{Ch}$ 103/2 were again generally of lower self-fertility, while $\mathrm{Cp}$ I $45 \mathrm{~S} 2 / 3$ was highest in this group, but taken as a whole the group showed no lower pod and seed set than the progenies from highly fertile $S_{0}$ plants.

Line Ch I 70 R 5/2 was continued in its entirety in $195^{8}$, five segregates representing each of the $S_{1}$ progeny from the previous year. Of

TABLE 5

Pod and seed setting in $S_{3}$ progenies of $C_{170} R_{5 / 2}$

\begin{tabular}{|c|c|c|c|c|}
\hline \multirow{3}{*}{ Progeny no. } & \multicolumn{3}{|c|}{$\mathrm{S}_{3}$ progeny } & \multirow{3}{*}{$\begin{array}{c}\text { No. of } \\
\text { plants } \\
\text { per } S_{3} \\
\text { progeny }\end{array}$} \\
\hline & \multicolumn{3}{|c|}{ Mean no. of } & \\
\hline & Pods & Seeds & Seeds per pod & \\
\hline $1 / 2$ & $3 \cdot I$ & $5 \cdot 4$ & $I \cdot 7$ & I 5 \\
\hline $4 / 1$ & $4 \cdot \mathrm{I}$ & 7.5 & $1 \cdot 8$ & 14 \\
\hline $7 / 2$ & $2 \cdot 2$ & 3.4 & $1 \cdot 5$ & 12 \\
\hline $9 / 1$ & $6 \cdot 4$ & $11 \cdot 6$ & $\mathrm{I} \cdot 8$ & I 5 \\
\hline $9 / 2$ & $9 \cdot 7$ & $18 \cdot 6$ & $1 \cdot 9$ & 12 \\
\hline $13 / 1$ & $\mathrm{I} \cdot 4$ & $2 \cdot 4$ & $1 \cdot 7$ & 3 \\
\hline $20 / 1$ & $4 \cdot 7$ & $10 \cdot 5$ & $2 \cdot 2$ & I 5 \\
\hline $21 / 22$ & I I 4 & 23.5 & $2 \cdot 1$ & II \\
\hline $21 / 1 \mathrm{D}$ & II $\cdot 6$ & $28 \cdot 2$ & $2 \cdot 4$ & 10 \\
\hline $10 / \mathrm{ID}$ & $6 \cdot 0$ & $12 \cdot 4$ & $2 \cdot I$ & 8 \\
\hline $24 / I$ & $2 \cdot 5$ & $4 \cdot 8$ & I.9 & I 5 \\
\hline \multirow{3}{*}{$29 / 1$} & $5 \cdot 4$ & $9 \cdot 9$ & I. 8 & 13 \\
\hline & $5 \cdot 6$ & $10 \cdot 0$ & $\mathrm{I} \cdot 8$ & 15 \\
\hline & $5 \cdot 7$ & II $\cdot 4$ & I 99 & $\cdots$ \\
\hline
\end{tabular}

the other lines, five plant progenies were sown of $\mathrm{Ch} 35 / 2 \mathrm{R}_{3 / 1}$ and Ch ${ }_{103} \mathrm{~S}_{2 / 4}$ and, in addition, five plant progenies (where seed permitted) of the highly self-sterile line $\mathrm{Ch}_{10} / \mathrm{R}_{3 / 2}$. Pod and seed number per plant were again recorded and the data for Ch $\mathrm{I}_{70} \mathrm{R}_{5 / 2}$ are given in table 4 .

Pod and seed number per plant are slightly improved in 1958 although no conscious selection has been practised for this factor. If, however, the $195^{8}$ population had been based only on those plants with 16 or more seeds, the means for pods and seeds would be $7 \cdot 3$ and 12.6 (compared with 5.9 and I I $\circ$ for unselected plants). In this family, selection would have improved self-fertility. In $\mathrm{Ch}_{35 / 2} \mathrm{R}_{3 / \mathrm{I}}$ and $\mathrm{Ch}_{103 / 2} \mathrm{~S}_{2 / 4}$ where some selection was in fact practised the means were maintained in the former ( 4.1 for pods and $7 \cdot 3$ for seeds) while they fell in $\mathrm{Ch}_{103 / 2} \mathrm{~S}_{2 / 4}$ (to 3.0 for pods and 6.0 for seeds). The low fertility line $\mathrm{Ch} \mathrm{I03/2}_{3 / 2}$ had a lower pod set (0.97 per 
plant) but maintained seed setting at $\mathrm{I} \cdot 6$ per plant, with again over 50 per cent. self-sterile plants.

The I959 material was based on progenies from $\mathrm{S}_{2}$ plants which bore over 20 seeds. $S_{3}$ progeny means for pods and seeds per plant and seeds per pod are given in table 5 .

TABLE 6

Pod and seed setting in parent plants, $S_{0}$ plants and $S_{1}$ progenies of $C h 4$

\begin{tabular}{|c|c|c|c|c|c|c|c|c|c|}
\hline \multirow[b]{2}{*}{$\begin{array}{l}\text { Line and } \\
\text { progeny } \\
\text { no. }\end{array}$} & \multicolumn{2}{|c|}{ Pod no. of } & \multicolumn{7}{|c|}{ Means of $S_{1}$ progenies (1957) } \\
\hline & $\begin{array}{l}\text { Parent } \\
\text { plants } \\
\text { (I955) } \\
\text { (open- } \\
\text { pollina- } \\
\text { tion) }\end{array}$ & $\begin{array}{c}S_{0} \\
\text { plants } \\
\text { (1956) } \\
\text { (tripping) }\end{array}$ & Flowers & Pods & Seeds & $\begin{array}{l}\text { Per } \\
\text { cent. } \\
\text { flowers } \\
\text { set }\end{array}$ & $\begin{array}{l}\text { Seeds } \\
\text { per } 100 \\
\text { flowers }\end{array}$ & $\begin{array}{c}\text { Per } \\
\text { cent. } \\
\text { flowers } \\
\text { set }\end{array}$ & $\begin{array}{l}\text { Seeds } \\
\text { per 10o } \\
\text { flowers }\end{array}$ \\
\hline $\begin{array}{r}\mathrm{Ch}_{4} \mathrm{R}_{\mathrm{I}} / \mathrm{I} \\
2 / \mathrm{I} \\
3 / \mathrm{I} \\
4 / \mathrm{I} \\
5 / 1\end{array}$ & $3^{I}$ & $\begin{array}{r}\mathrm{I} 3 \\
6 \\
\mathrm{II} \\
6 \\
9\end{array}$ & $\begin{array}{l}108 \cdot 2 \\
102 \cdot 0 \\
100 \cdot 2 \\
101 \cdot 0 \\
107 \cdot 0\end{array}$ & $\begin{array}{r}5 \cdot 4 \\
2 \cdot 0 \\
3 \cdot 0 \\
14 \cdot 0 \\
4^{\cdot} \cdot 2\end{array}$ & $\begin{array}{r}9 \cdot 0 \\
3 \cdot 0 \\
5 \cdot 2 \\
36 \cdot 5 \\
7 \cdot 0\end{array}$ & $\begin{array}{r}5 \cdot 0 \\
2 \cdot 0 \\
3 \cdot 0 \\
13 \cdot 9 \\
3 \cdot 9\end{array}$ & $\begin{array}{r}8 \cdot 2 \\
3 \cdot 2 \\
5 \cdot 3 \\
36 \cdot 1 \\
6 \cdot 3\end{array}$ & $4 \cdot 4$ & $8 \cdot 5$ \\
\hline $\begin{array}{l}1 / 2 \\
2 / 2 \\
3 / 2 \\
4 / 2 \\
5 / 2\end{array}$ & 24 & $\begin{array}{r}2 \\
5 \\
10 \\
2 \\
10\end{array}$ & $\begin{array}{r}109 \cdot 0 \\
72 \cdot 0 \\
100 \cdot 8 \\
101 \cdot 4 \\
85 \cdot 4\end{array}$ & $\begin{array}{l}4 \cdot 0 \\
\mathrm{I} \cdot 8 \\
0.6 \\
\mathrm{I} \cdot 2 \\
0.8\end{array}$ & $\begin{array}{l}6 \cdot 0 \\
3 \cdot 0 \\
1 \cdot 6 \\
1 \cdot 2 \\
1 \cdot 6\end{array}$ & $\begin{array}{l}3 \cdot 7 \\
2 \cdot 5 \\
0 \cdot 6 \\
1 \cdot 2 \\
1 \cdot 0\end{array}$ & $\begin{array}{l}4 \cdot 8 \\
3 \cdot 8 \\
1 \cdot 5 \\
1 \cdot 3 \\
1 \cdot 9\end{array}$ & I 6 & $2 \cdot 5$ \\
\hline $\begin{array}{l}1 / 3 \\
4 / 3\end{array}$ & 24 & $\begin{array}{r}8 \\
10\end{array}$ & $\begin{array}{l}106 \cdot 0 \\
106.8\end{array}$ & $\begin{array}{l}0 \cdot 6 \\
3 \cdot 4\end{array}$ & $\begin{array}{l}1 \cdot 0 \\
7 \cdot 2\end{array}$ & $\begin{array}{l}0.6 \\
3.2\end{array}$ & $\begin{array}{l}0.9 \\
6 \cdot 9\end{array}$ & $\mathrm{I} \cdot 9$ & $3 \cdot 9$ \\
\hline $\begin{array}{l}1 / 4 \\
2 / 4 \\
3 / 4 \\
4 / 4 \\
5 / 4\end{array}$ & I5 & $\begin{array}{l}17 \\
20 \\
16 \\
13 \\
6\end{array}$ & $\begin{array}{r}98 \cdot 4 \\
104 \cdot 6 \\
92 \cdot 6 \\
109 \cdot 0 \\
106 \cdot 3\end{array}$ & $\begin{array}{r}6 \cdot 6 \\
5 \cdot 2 \\
4 \cdot 0 \\
10 \cdot 4 \\
9 \cdot 3\end{array}$ & $\begin{array}{r}9 \cdot 0 \\
7 \cdot 0 \\
6 \cdot 2 \\
15 \cdot 2 \\
17 \cdot 0\end{array}$ & $\begin{array}{l}6 \cdot 7 \\
4 \cdot 0 \\
4 \cdot 3 \\
9 \cdot 5 \\
8 \cdot 7\end{array}$ & $\begin{array}{r}9 \cdot 1 \\
5 \cdot 8 \\
6 \cdot 8 \\
14 \cdot 4 \\
15 \cdot 9\end{array}$ & $6 \cdot 7$ & 13.5 \\
\hline $\begin{array}{l}1 / 5 \\
2 / 5 \\
3 / 5 \\
4 / 5 \\
5 / 5\end{array}$ & $2 \mathrm{I}$ & $\begin{array}{r}5 \\
6 \\
7 \\
12 \\
8\end{array}$ & $\begin{array}{l}\text { IOI.6 } \\
\text { I } 36.6 \\
\text { I03.4 } \\
\text { I } 10.6 \\
\text { 100.8 }\end{array}$ & $\begin{array}{l}I \cdot 4 \\
4 \cdot 8 \\
3 \cdot 6 \\
I \cdot 0 \\
4 \cdot 6\end{array}$ & $\begin{array}{l}2 \cdot 0 \\
4 \cdot 0 \\
6 \cdot 2 \\
2 \cdot 0 \\
9 \cdot 0\end{array}$ & $\begin{array}{r}1.4 \\
3.5 \\
\cdot 3.5 \\
0.9 \\
4.6\end{array}$ & $\begin{array}{l}2 \cdot 0 \\
3 \cdot 1 \\
6 \cdot 1 \\
1 \cdot 8 \\
9 \cdot 0\end{array}$ & $2 \cdot 8$ & 4.4 \\
\hline Mean & $23 \cdot 0$ & $9 \cdot 2$ & $102 \cdot 9$ & $3 \cdot 8$ & $6 \cdot 4$ & $4^{\circ} \mathrm{O}$ & $7 \cdot 0$ & $\ldots$ & $\ldots$ \\
\hline
\end{tabular}

It will be observed that the mean number of pods, seeds, and seeds per pod, for the whole Ch I yo population has not been improved by the particular form of selection practised. However, two families stand out as consistently high pod and seed setters, namely, 9 and 2 I (tables 4,5 ), and of these No. $2 \mathrm{I}$ is extremely promising, but neither family could have been recognised in $S_{1}$ or $S_{2}$ as likely to give consistently self-fertile progeny.

It would appear that no general correlation exists between individual plants and their progenies with respect to pod and seed number. 


\section{INBREEDING OF $\mathrm{CH} 4$}

The original field population was analysed as fully as possible in I955. Forty plants within the population were marked at random and the numbers of flowers and pods were recorded throughout the season.

TABLE 7

Analysis of variance of differences between lines and also between progenies with respect to seeds per 100 flowers

\begin{tabular}{|c|c|c|c|c|c|}
\hline Component & & $\mathbf{N}$ & Mean squares & $\mathrm{F}$ & Probability \\
\hline \multirow[t]{2}{*}{$\begin{array}{l}\text { Between lines } \\
\text { Between progenies } \\
\text { Within progenies }\end{array}$} & . & $\begin{array}{r}4 \\
22 \\
76\end{array}$ & \multirow[t]{2}{*}{$\begin{array}{r}242 \cdot 0 \\
153.8 \\
12.1\end{array}$} & \multirow[t]{2}{*}{$\begin{array}{c}20 \cdot 1 \\
12 \cdot 7 \\
\cdots\end{array}$} & \multirow[t]{2}{*}{$\begin{array}{cl}<0.1 & \text { per cent } \\
<0.1 & \text { per cent } \\
& \ldots\end{array}$} \\
\hline & & 102 & & & \\
\hline
\end{tabular}

TABLE 8

Pod and seed setting in $S_{1}$ progeny plants, $S_{2}$ families and $S_{3}$ families of Ch 4

\begin{tabular}{|c|c|c|c|c|c|c|c|c|}
\hline \multirow{3}{*}{$\begin{array}{l}\text { Progeny and } \\
\text { family no. }\end{array}$} & \multicolumn{2}{|c|}{$\begin{array}{l}\mathrm{S}_{1} \text { plants } \\
(1957)\end{array}$} & \multicolumn{3}{|c|}{$\begin{array}{c}\mathrm{S}_{2} \text { families } \\
\left(195^{8}\right)\end{array}$} & \multicolumn{3}{|c|}{$\begin{array}{c}\mathrm{S}_{\mathbf{3}} \text { families } \\
\text { (I959) }\end{array}$} \\
\hline & \multicolumn{2}{|c|}{ No. of } & \multicolumn{3}{|c|}{ Mean no. of } & \multicolumn{3}{|c|}{ Mean no. of } \\
\hline & Pods & Seeds & Flowers & Pods & Seeds & Flowers & Pods & Seeds \\
\hline $\begin{array}{r:r}4 & (1) \\
& (2)\end{array}$ & $\begin{array}{l}13 \\
15\end{array}$ & $\begin{array}{l}35 \\
3^{8}\end{array}$ & $\begin{array}{l}59 \cdot 4 \\
67 \cdot 2\end{array}$ & $\begin{array}{l}3 \cdot 7 \\
5 \cdot 3\end{array}$ & $\begin{array}{r}9.5 \\
11.9\end{array}$ & $\begin{array}{l}71 \cdot 1 \\
55 \cdot 0\end{array}$ & $\begin{array}{l}4 \cdot 5 \\
8 \cdot 0\end{array}$ & $\begin{array}{r}9 \cdot 5 \\
18.5\end{array}$ \\
\hline $\begin{array}{l}12(2) \\
13\end{array}$ & $\begin{array}{r}2 \\
10\end{array}$ & $\begin{array}{r}3 \\
15\end{array}$ & $\begin{array}{l}203.7 \\
135.6\end{array}$ & $\begin{array}{l}1 \cdot 7 \\
4 \cdot 3\end{array}$ & $\begin{array}{l}2 \cdot 7 \\
7 \cdot 4\end{array}$ & $\begin{array}{l}83 \cdot 0 \\
63 \cdot 8\end{array}$ & $\begin{array}{l}0.8 \\
3 \cdot 6\end{array}$ & $\begin{array}{l}1 \cdot 3 \\
7 \cdot 0\end{array}$ \\
\hline $44(5)$ & 14 & 18 & 111.9 & $4 \cdot 8$ & $9^{\cdot I}$ & $65 \cdot 2$ & 5.9 & $10 \cdot 1$ \\
\hline $54(4)$ & 19 & 30 & $90 \cdot 1$ & $2 \cdot 9$ & $4 \cdot 6$ & $67 \cdot 7$ & $3 \cdot 2$ & 4.9 \\
\hline $25(3)$ & 8 & II & $75 \cdot 2$ & $4 \cdot 8$ & $8 \cdot 8$ & $54 \cdot 5$ & $4 \cdot 9$ & $9^{\cdot 1}$ \\
\hline Mean & $11 \cdot 6$ & $21 \cdot 4$ & $106 \cdot 2$ & $3 \cdot 9$ & $7 \cdot 7$ & $65 \cdot 8$ & $7 \cdot 7$ & $8 \cdot 6$ \\
\hline
\end{tabular}

The number of flowers per plant varied from 50-I50 with a mean of $98 \cdot 0$. The pods had a much more restricted distribution, namely, from 5-50 per plant with a mean of $23^{\circ} \mathrm{I}$. Between Io and 50 per cent. of the total flowers set pods, the mean flowers set being 23.6 per cent.

Progenies were grown from each of the five random plants described in table $\mathrm{I}$ and allowed to set seed after tripping in an insect-proof glasshouse. The data on selfing this $\mathrm{Ch} 4$ population are given in table 6 . 
Pod number although reduced in most $\mathrm{S}_{0}$ plants was still high in some, particularly in those derived from $\mathrm{Ch}_{4} \mathrm{R}_{4}$. In 1957 , random progenies were grown from each original line and they were allowed to set seed unaided in an insect-proof glasshouse. Pod number was still further reduced, except in $\mathrm{Ch}_{4} \mathrm{R}_{4}$ progenies and in line $4 / \mathrm{I}$ where a considerable increase occurred. An analysis of the differences between progenies and lines on the basis of seeds set per roo flowers is given in table 7 .

Lines $\mathrm{Ch}_{4} \mathrm{R}_{\mathrm{I}}$ and $\mathrm{Ch}_{4} \mathrm{R}_{4}$ are significantly better than the others and differences between progenies within lines are also significant, progenies $4 / \mathrm{I}, 4 / 4$ and $5 / 4$ being particularly self-fertile.

Progenies $4 / \mathrm{I}, \mathrm{I} / 2,2 / 4,4 / 4,5 / 4$ and $2 / 5$ were continued in $195^{8}$ and 1959 , and all the available seed was sown up to a maximum of 20 per progeny. These plants were again allowed to set seed unaided in an insect-proof glasshouse; the number of flowers, pods and seeds per plant were recorded (table 8).

The mean numbers of pods and seeds again increased in 1959, also the percentage of flowers forming pods. This increased flower setting cannot be due to the elimination of self-sterile plants, as can be seen from the following figures:

\begin{tabular}{|c|c|c|c|}
\hline Year & Generation & $\begin{array}{l}\text { Per cent. } \\
\text { flowers setting pods }\end{array}$ & $\begin{array}{l}\text { Per cent. } \\
\text { sterile plants }\end{array}$ \\
\hline $\begin{array}{l}1955 \\
1956 \\
1957 \\
1958 \\
1959\end{array}$ & $\begin{array}{l}\text { Open-pollinated parents } \\
\mathbf{S}_{0} \text { selfed plants (tripped) } \\
\mathbf{S}_{1}, ", ", \\
\mathbf{S}_{2}, ", ", \\
\mathbf{S}_{3}, ",\end{array}$ & $\begin{array}{r}23 \cdot 6 \\
7 \cdot 3 \\
3 \cdot 7 \\
5 \cdot 1 \\
1 x \cdot 7\end{array}$ & $\begin{array}{r}0 \cdot 0 \\
5 \cdot 6 \\
19 \cdot 4 \\
2 \cdot 1 \\
10 \cdot 4\end{array}$ \\
\hline
\end{tabular}

Although the reduced number of flowers per plant in I 959 may be partly responsible for the relative increase in flower setting, nevertheless this improvement is a real effect and not a chance occurrence. This is shown by the analysis of the correlations of $S_{3}$ on $S_{2}$ family means for number of pods and seeds per plant. For pods $r=+0.9 \mathrm{I}$ and for seeds $r=0.94$, both correlation coefficients are highly significant $(\mathbf{P}=0 \cdot \mathrm{I}$ per cent). Not only do the families and progenies differ significantly from each other in self-fertility but these differences appear to be inherited, and therefore are capable of improvement by selection. Some improvement in seed number per plant is shown but no conscious selection has been made for seed-setting on selfing. In fact it would appear that as soon as direct selection is made for seed-setting (as in Ch I 70) the actual seed-set may be reduced.

\section{DISCUSSION}

The behaviour of the two populations $\mathrm{Ch}_{4}$ and $\mathrm{Ch}$ I 70 under inbreeding offers an interesting comparison. The families were grown alongside each other every year and the only difference between them 
was that Ch 4 was based on unselected progenies in each generation while the Ch $\mathrm{I}$ 70 population was subjected to intensive selection for high seed-set:

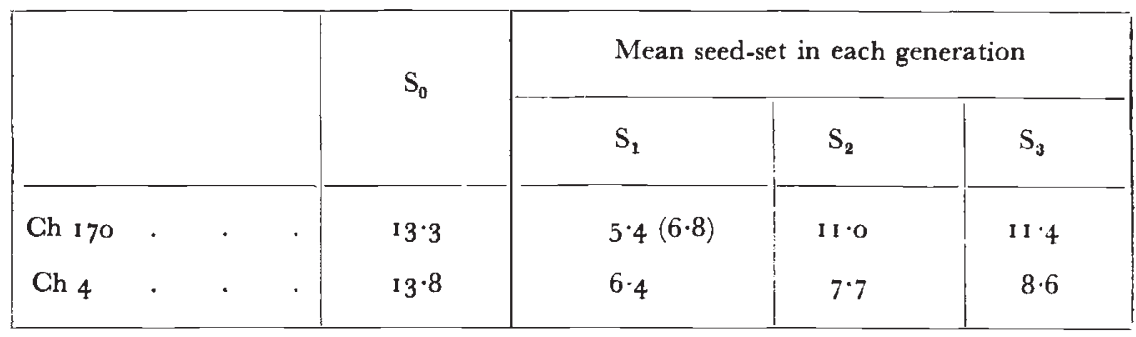

The flowers in the $\mathrm{S}_{0}$ generation were hand "tripped" and this accounts for the larger seed-set. In the $S_{1}$ generation the highlyselected Ch I 70 progenies had a lower seed-set $(5 \cdot 4)$ than those where selection had been relaxed $(6 \cdot 8$ seeds). Despite the intensive selection seed-set was only maintained in the $\mathrm{S}_{3}$ generation of $\mathrm{Ch}$ I 70 while the unselected $\mathrm{Ch} 4$ progenies continued to improve in seed-set.

Genetic variability for seed-setting on selfing is undoubtedly present in the material since the lines here diverged on inbreeding (table 7) although the grand mean has remained little changed throughout. Sybenga (1958) has described an experiment in rye where a record was made of seed-setting on self-pollination during inbreeding. Seedset improved in $S_{2}$ and $S_{3}$ generation but decreased in the $S_{4}$ and $S_{5}$ generations. Moreover, seed-set on selfing never exceeded that set after free pollination and only approached it in the $S_{3}$. Similar situations were observed on inbreeding grasses and other forage crops (Wexelsen, 1952) and particularly in red clover (Thomas, 1955).

Thompson and Rees (1956) demonstrated that during inbreeding of rye some selection occurred for heterozygotes. Selection did not operate in favour of heterozygotes in general, but in favour of a particular heterozygous combination, indicating that heterozygosity in itself is not necessarily advantageous. It was not possible to rule out completely a recessive gene of major effect, but it was suggested that a more likely explanation was that of a superior balance of polygenes in that particular hybrid combination.

In part I of the present investigation (Rowlands, r96o) infertility at the zygotic level was explained in terms of homozygosis of deleterious recessive genes following inbreeding, and, moreover, that these genes were linked together in a balanced system. It is inevitable with only six linkage groups that other balanced combinations will be associated with these linked deleterious recessives and will contribute to the superiority of certain heterozygous combinations. The selective advantage in natural populations of the linkage within and between polygenic systems has been discussed by Mather (1953). Correlated responses to selection can be extensive under such conditions and can 
give rise to true inertia (or homeostasis) but although they slow down, they do not finally prevent response to selection.

Evidence for dominance and non-allelic interaction of the genes controlling self-fertility was obtained from the diallel analysis of seedsetting in part I, and Jinks (1955) has related similar genetic situations in maize and other crops to close linkage in opposition of particular gene combinations. In such a system, inbreeding will fix the balanced combinations (Mather, I953) and since the phenotypes determined by the different balanced combinations are alike they will be retained equally in the population. Effective segregation and recombination cannot occur in the absence of crossing, so that the balanced combinations will not be broken up despite the presence of genic differences. When the variability has been frozen in this way, change under directional selection is impossible and recombination must be induced by crossing over for any progress to take place in the population.

Breese (1956) has discussed assortative (i.e. like by like) mating in this context and concludes that "where genes controlling a character tend to be linked in opposition, assortative mating followed by selection, by avoiding the premature homozygosis and fixation resulting from inbreeding should maximise the chances of securing and ultimately fixing desirable recombinants". Such a programme of assortative mating and selection in beans might well result in the isolation of vigorous self-fertile lines in which a new internal chromosomal balance may be established.

The data presented by Williams and Gilbert (1960) show that such internal balance has been achieved unconsciously in the tomato during its change-over from outbreeding to inbreeding. Consequently, once the underlying genetic system has been discovered it is reasonable to suppose that a properly designed breeding programme should be able to complete quickly and effectively the change-over to inbreeding that may have begun naturally in any outbreeding species.

\section{SUMMARY}

I. The behaviour of pod and seed-set under self-pollination has been investigated during a programme of inbreeding.

2. Self-pollination was relatively ineffective in producing seeds, and selection for this character over three generations of inbreeding was not successful. Intensive selection for seed-set on self-pollination in one population had no marked effect, while in an unselected population seed-set appeared to improve slightly after three generations.

3. It is suggested that the presence of deleterious recessive genes, coupled with the development of close linkage in opposition of particular gene combinations, has produced a balanced system which directional selection alone is unable to break up.

4. Assortative mating followed by selection, by avoiding the fixation resulting from inbreeding, should maximise the chances of securing 
and fixing desirable recombinants, and such a programme is recommended for the development of vigorous self-fertile lines not only in field beans but also in other outbreeding crops.

Acknowledgments.-I should like to thank Professor P. T. Thomas, Director of the Welsh Plant Breeding Station, and other colleagues for advice and helpful criticism during the investigation.

\section{REFERENCES}

BREESE, E. L. 1956. The genetical consequences of assortative mating. Heredity, Io, 323-343.

JiNks, J. L. 1955. A survey of the genetical basis of heterosis in a variety of diallel crosses. Heredity, 9, 223-238.

MATHER, K. 1953. The genetical structure of populations. Symp. Soc. exp. Biol., 7 (Evolution), 66-95.

ROWLANDS, D. G. 1958 . The nature of the breeding system in the field bean ( . faba

L.) and its relationship to breeding for yield. Heredity, I2, 1 13-126.

Rowlands, D. G. 1960. Fertility studies in the field bean (Vicia faba L.). I. Crossand self-fertility. Heredity, $15, \mathrm{I} 6 \mathrm{I}-\mathrm{I} 73$.

SYBENGA, J. 1958. Inbreeding effects in rye. Z. fur Vererb.-Lehr., 89, 338-354.

тномаs, н. L. 1955. Inbreeding and selection of self-fertilised lines of red clover, Trifolium pratense. Agron. F., 47, 487-489.

THOMPSON, J. B., AND REES, н. 1956. Selection for heterozygotes during inbreeding. Nature, Lond., $177,385-386$.

wexelsen, H. 1952. The use of inbreeding in forage crops. Proc. 6th Int. Grass. Congr., I, 299-305.

Williams, W., AND Gilbert, N. I960. Heterosis and the inheritance of yield in the tomato. Heredity, 14, 133-149. 